rev Psi

Revista de Psicología (UNLP)

https://revistas.unlp.edu.ar/revpsi
Reseña de evento científico

\section{Segundo Congreso Internacional de Victimología: "Intervenciones sobre las violencias. Nuevos desafíos: de la multidisciplina a los inter-saberes"}

\author{
Ana Clara D’0vidio ${ }^{1}$ \\ Correspondencia \\ anita_dovidio@hotmail.com \\ Filiaciones institucionales \\ ${ }^{1}$ Facultad de Psicología, Universidad Nacional de \\ La Plata (Argentina)
}

Xavier Oñativia ${ }^{1}$

\section{Datos del evento}

El Segundo Congreso Internacional de Victimología: "Intervenciones sobre las violencias. Nuevos desafíos: de la multidisciplina a los inter-saberes" se llevó a cabo los días 26, 27 y 28 de septiembre del año 2018, en la Facultad de Psicología de la Universidad Nacional de La Plata (UNLP).

El Congreso constituyó un evento académico de excelencia, en el que participaron más de 1600 personas, profesionales y estudiantes de Psicología, Trabajo Social, Ciencias Jurídicas, Medicina, Comunicación Social, Educación, Seguridad, entre otras áreas de conocimiento, con la participación de diversas organizaciones de la comunidad. Asistentes de diversos lugares, tanto de numerosas provincias de nuestro país como del exterior, se dieron cita en el evento. Se resalta la presencia de asistentes e invitados/as internacionales de ocho países: Paraguay, Colombia, Brasil, México, Uruguay, Venezuela, Ecuador y Chile que posibilitaron pensar la victimología en clave latinoamericana, uno de los objetivos del Congreso.
Editor

Nicolás Alessandroni | Facultad de Psicología, Universidad Autónoma de Madrid (España)
D'Ovidio, A. C. y Oñativia, X. (2018).

Segundo Congreso Internacional de Victimología: "Intervenciones sobre

las violencias. Nuevos desafíos: de multidisciplina a los inter-saberes". Revista de Psicología, 17(2), 82-88. doi: $10.24215 / 2422572 \mathrm{Xe} 023$.
ISSN

2422-572X

Licencia

(c) Copyright: D'Ovidio, A.C. y Oñativia, X. Se distribuye bajo una licencia de Cultura Libre CC-BY 4.0

Entidad editora

RevPsi es una publicación de la

Facultad de Psicología (Universidad

Nacional de La Plata, Argentina)

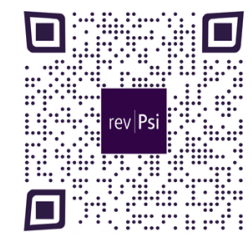

ACCESO ABIERTO DIAMANTE ə 
El encuentro se desarrolló en un clima de entusiasmo y camaradería, aspecto que a lo largo de las distintas ediciones se ha convertido en un rasgo distintivo de esta convocatoria. Ello genera las condiciones para propiciar el debate y la profundización de saberes en torno al estudio de las complejidades que presenta el abordaje de las violencias, caracterizado por la participación activa, desde una perspectiva crítica y con especial interés en la producción de conocimiento situado.

\section{Contexto}

El Segundo Congreso Internacional de Victimología tiene como antecedentes diversas actividades y encuentros que se vienen desarrollando desde el año 2009 en la Facultad de Psicología de la Universidad Nacional de La Plata. Entre ellas, se resaltan el dictado del Seminario Optativo sobre Victimología entre los años 2009 y 2016, la realización de cinco Jornadas Nacionales, así como los valiosos intercambios generados en el Primer Congreso Nacional de Victimología, llevado a cabo en el año 2013, y el Primer Congreso Internacional en el año 2016.

En esta oportunidad el evento contó con el auspicio y acompañamiento de reconocidas instituciones de nuestro país: la Asociación Abuelas de Plaza de Mayo, la Asociación Madres del Dolor, Colegios Profesionales de la República Argentina, cuerpos legislativos locales y provinciales, entre otros.

A lo largo de los tres días en que se llevó a cabo el Congreso, licenciados/as y doctores/as en psicología, trabajadores/as sociales, magistrados/as, juristas, médicos/ as psiquiatras entre otros/as, disertaron en conferencias, paneles interdisciplinarios y simposios, aportando valiosos insumos para reflexionar sobre las prácticas interdisciplinarias actuales en el campo victimológico. Entre las múltiples temáticas abordadas, se destacan las siguientes: violencias y derechos humanos, trata de personas, violencia contra las mujeres, abuso sexual infantil, maltrato infantil, violencias en instituciones de encierro, terrorismo de Estado, dispositivos de intervención para la asistencia a víctimas de violencias, políticas públicas en prevención de las violencias, género y diversidad, salud mental y derechos humanos, violencia institucional, medios de comunicación y subjetividad, violencias y acceso a la justicia, desempleo, exclusión social y subjetividad.

\section{Descripción y valoración}

En este encuentro académico se propuso el desafío de pensar lo interdisciplinario en una doble vía: como una necesidad operativa para la intervención sobre las violencias, y a la vez una como relectura crítica incorporando la idea de "intersaberes", conocimientos válidos más allá de los saberes disciplinares, que se ponen en juego en las intervenciones y prácticas del vasto campo de la victimología.

El evento constituyó un espacio para el encuentro, el debate, la profundización y actualización de saberes y prácticas acerca de las violencias en el campo victimológico, analizando el rol técnico profesional desde una posición ética y política comprometida 
con la perspectiva de Derechos Humanos.

Como actividades destacadas debe señalarse la realización de dos conferencias magistrales y tres paneles centrales, con destacados referentes nacionales e internacionales que abordaron temáticas de actualidad generando gran interés en el público asistente.

En el transcurso de los tres días se llevaron a cabo 14 espacios destinados al debate e intercambio bajo la modalidad de simposios por invitación, integrados por grupos de expertos/as en variadas áreas del campo de la victimología. Aquí participaron un total de 46 destacados/as panelistas, referentes nacionales e internacionales con experiencia en investigación, actividades de producción teórica y ejercicio profesional en diversos ámbitos referidos a las violencias.

Uno de los aspectos relevantes del evento fue la presentación de 91 comunicaciones bajo la modalidad de trabajos libres. Los mismos fueron agrupados de acuerdo a los ejes temáticos en 22 mesas que se llevaron a cabo durante los tres días del Congreso. De esta forma 215 expositores/as nacionales e internacionales tuvieron la posibilidad de presentar sus producciones escritas referidas al amplio campo de las problemáticas concernientes a los efectos de las violencias en el sujeto, sus grupos de pertenencia y en la comunidad, tanto en lo referido a los procesos de victimización, revictimización como de desvictimización, experiencias de intervenciones, avances de investigación e investigaciones finalizadas.

Asimismo, en el marco de Congreso se llevaron a cabo otras actividades entre las que se destacan la exposición de pósteres y el espacio para la presentación de cinco libros: Territorios adolescentes y entretiempo de la sexuación (Comps. Adrián Grassi y Néstor Córdova, Editorial EntreIdeas, 2018), Huellas. Después de la muerte de un hijo (Silvia Irigaray, Editorial Planeta, 2017), Masculinidades y mandatos del patriarcado neoliberal. Una lectura psicoanalítica con perspectiva de género (Marta Fernández Boccardo y María Laura Peretti, Editorial EntreIdeas, 2018), Aborto. Aspectos normativos, jurídicos y discursivos (Coord. Daniel Busdygan, Editorial Biblos, 2018), y Mariposas libres. Derecho a vivir una infancia trans (Gabriela Mansilla, Editorial Universidad Nacional de General Sarmiento, 2018). De esta forma, los/as autores/as tuvieron la oportunidad de presentar sus más recientes producciones, dando lugar a interesantes y fructíferos intercambios con el público asistente.

Cabe mencionar también la realización del II Encuentro Internacional de Estudiantes de Psicología, donde estudiantes de nuestra Facultad y de otras Facultades del país y del exterior -Uruguay, México, Colombia, Francia y Brasil- se dieron cita para debatir e intercambiar experiencias sobre la formación específica en el campo de la psicología para intervenir sobre las violencias, su prevención, abordaje y erradicación, bajo la consigna "Pensar nuestra formación para intervenir sobre las violencias".

El inicio del Congreso estuvo a cargo del Vicepresidente del Área Académica de la Universidad Nacional de La Plata Lic. Martín López Armengol y del Decano de la Facultad de Psicología Lic. Xavier Oñativia, quienes resaltaron la importancia 
académica que reviste la realización de eventos científicos de tal envergadura. Al mismo tiempo, se destacaron los aportes de las ediciones anteriores -Congresos y Jornadas de Victimología-, señalando la relevancia de llevar adelante desde la Universidad Pública iniciativas referidas a investigación y prácticas con compromiso social, enmarcadas en el paradigma de los derechos humanos.

A continuación tuvo lugar la Conferencia Magistral de Apertura "De los procesos de vulnerabilización a la experiencia de la multitud: potencias colectivas, corporalidades, subjetividades en acción" a cargo de la Dra. en Psicología, profesora consulta e investigadora de la Universidad de Buenos Aires, Ana María Fernández, quien disertó ante un auditorio de más de 500 personas.

Esta presentación caracterizó críticamente aspectos de la sociedad patriarcal y analizó diversas modalidades de reacción social por parte de movimientos encabezados por mujeres y disidencias sexuales. Puso en valor la potencialidad presente en las distintas modalidades de respuesta organizadas de manera colectiva, ejemplificando con experiencias recogidas en distintos lugares del país y del mundo. Se resaltó la creatividad, el dinamismo, la originalidad y la fuerza social creciente con que movimientos sociales impulsados por mujeres y disidencias sexuales afrontan la violencia patriarcal y propician modificaciones estructurales en la organización social.

Como ya se señaló tuvieron lugar tres paneles centrales. Uno de ellos, "Victimología desde una perspectiva latinoamericana. Recorridos históricos y desafíos actuales" propuso pensar la particularidad de generar teorías, prácticas e intervenciones en el campo del abordaje de las violencias desde nuestro contexto latinoamericano, tomando como eje referencial la descolonización de la producción de conocimiento. En este panel se contó con la participación del psicólogo y representante del Instituto Latinoamericano de las Naciones Unidas para la Prevención del Delito y la Justicia Penal (ILANUD) Juan Carlos Domínguez Lostaló, el psicólogo y creador del primer Centro de Asistencia a Víctimas de Atentados Sexuales de Chile, Elías Escaff Silva, y el docente de la Escuela Superior y del Centro de Altos Estudios en Especialidades Policiales de la Policía de la Provincia de Buenos Aires, comisario inspector retirado Raúl Cheves.

En primer lugar Elías Escaff Silva situó conceptos clave para la delimitación del corpus teórico victimológico actual, recuperando aportes de autores latinoamericanos y analizando distintas consideraciones que conviven en la amplitud de enfoques. Además puso de relieve el interés central de las personas víctimas de violencia: la búsqueda de justicia, resaltando la necesidad de orientar la asistencia hacia ese objetivo. Posteriormente Raúl Cheves analizó la relación de la victimología con uno de los aspectos centrales que la atraviesan: el rol de las fuerzas de seguridad. Caracterizó su funcionamiento a partir de lo que ha postulado como modelo policial hegemónico, y su impacto en la relación con la población. Profundizó su análisis articulando con modelos de seguridad presentes en diversos pueblos originarios precolombinos, puntualizando sus distintas prioridades y objetivos generales. 
A su turno Juan Carlos Domínguez Lostaló presentó un recorrido que integró tanto aspectos conceptuales como históricos, poniendo énfasis en los procesos de violentamiento que Latinoamérica ha sufrido desde la conquista europea. Resaltó los procesos de victimización sobre la que se ha forjado el continente americano y la importancia de la relación pasado-presente basada en la verdad y justicia, y su relevancia para la salud mental de la población.

El segundo panel central se denominó "Infancias trans: nuevos existenciarios y derecho a la identidad" y abordó por primer vez este aspecto de la temática en la Facultad de Psicología de la Universidad Nacional de La Plata. Estuvo a cargo de la Dra. Débora Tajer, el psicoanalista Facundo Blestcher y la Sra. Gabriela Mansilla -ONG Infancias Libres- quienes abordaron las transformaciones socio-históricas, culturales, políticas y legislativas actuales, poniendo de relieve la diversidad de experiencias y recorridos que demarcan nuevos posibles existenciarios.

Débora Tajer y Facundo Blestcher interpelaron teorías y prácticas instituidas así como posicionamientos de ciertos/as psicoanalistas frente a la compleja temática de la construcción de la subjetividad y constitución del psiquismo en aquellos/as niños/ as que tempranamente manifiestan una no correspondencia entre sexo biológico e identidad de género. En sus desarrollos, buscaron poner en diálogo el psicoanálisis con las teorías de género, para pensar las subjetividades contemporáneas desde un posicionamiento ético y profesional a la altura de los desafíos de época, poniendo de relieve que una niñez trans es una infancia posible.

Por su parte Gabriela Mansilla, madre de Luana, primera niña transgénero del mundo que obtuvo su DNI acorde a su identidad de género autopercibida sin instancia judicial, habló de su lucha por el derecho a la identidad de su hija. Como activista política referente de la defensa de los derechos de las infancias trans, refirió el apoyo que brinda desde la institución que dirige a familias que atraviesan situaciones similares a la suya. Su participación enriqueció el debate, a partir de aportar las vicisitudes que ha debido sortear a lo largo de los años a causa de la falta de información de profesionales, instituciones y organismos estatales, en una sociedad que aun hoy presenta resistencias a la hora de pensar que la identidad no se funda en la genitalidad, sino que se trata de una construcción sociocultural de los géneros.

Del mismo modo, numerosa concurrencia tuvo el panel interdisciplinario "Interrupción voluntaria del embarazo: obstáculos y posibilidades para un cambio cultural', conformado por el médico psicoanalista Juan Carlos Volnovich, la militante social, sindical y feminista Estela Díaz, la psicóloga Edith Pérez Secretaria de Salud de la UNLP, y la referente del periodismo con perspectiva de género, Mariana Carbajal.

Edith Pérez señaló que la lucha por el aborto legal, seguro y gratuito permite instalar en la agenda social, histórica y política las bases para nuevas producciones subjetivas en torno a la maternidad y la sexualidad. Esta mirada exige revisar las teorías para la formación de profesionales, ciudadanos y ciudadanas dignos de su tiempo, que puedan interrogarse a sí mismos/as, interrogar su momento y sus significaciones. Solo eso permitirá salirse de dogmas de todo tipo y transformar a las nuevas generaciones. 
Mariana Carbajal subrayó la importancia de la despenalización social del aborto, a partir del estado público que tomó el debate. Como saldo positivo del mismo destacó la organización de mujeres y el armado de redes de asistencia y contención, la instalación del tema de la separación iglesia-Estado por las consecuencias que tiene en la política y en la vida de la población, la visibilización de que el aborto clandestino se cobra vidas de las mujeres de sectores más vulnerables y la construcción de consenso en torno a la necesidad de trabajar la educación sexual integral como herramienta para un cambio social.

Por su parte Estela Díaz señaló que si bien la Campaña por el Derecho al Aborto plantea una ley, lo que está de fondo es un cambio cultural, en relación a la autonomía y ciudadanía de las mujeres. Es por esto que se puede hablar de un triunfo político por más que no haya sido sancionada, ya que permitió sacar de la clandestinidad la palabra aborto e instaurar el diálogo intergeneracional de un tema prohibido. En ese sentido puso en valor el fuerte movimiento social de las mujeres en Argentina, en confrontación a las desigualdades, machismo y patriarcado.

Juan Carlos Volnovich planteó la importancia de separar la sexualidad de la reproducción, distinguiendo la capacidad de procrear de la obligación de hacerlo. Además afirmó que la penalización del aborto supone que las mujeres no son dueñas de su cuerpo y los abortos clandestinos cobran una innumerable cantidad de vidas que podrían evitarse legalizándolo. También puntualizó que al hablar de aborto se presenta el desafío de superar, tanto en la teoría como en la práctica política, el dominio de la sexualidad naturalizada, el imperativo de la heterosexualidad procreativa y la hegemonía del discurso posestructuralista para hacer propia la consigna: educación sexual para decidir, anticonceptivos para no abortar y aborto legal para no morir.

El cierre del Congreso estuvo a cargo de la Dra. en Antropología Rita Segato, investigadora de nivel máximo del Consejo Nacional de Investigaciones Científicas y Tecnológicas de Brasil, quien, ante un Salón Auditorio colmado, presentó su conferencia magistral "Colonialidad, mandato de masculinidad y la catástrofe de género en América Latina".

La Dra. Rita Segato centró su disertación en sus últimos desarrollos teóricos en torno a la problemática de las violencias ejercidas contra todo aquello que desafía la jerarquía patriarcal, introduciendo la idea de que nos encontramos frente a una verdadera catástrofe de género en Latinoamérica. Basándose en sus aportes sobre lo que ha conceptualizado como la pedagogía de la crueldad, Segato invitó a pensar las relaciones de género y el sistema patriarcal imperante, poniendo énfasis en el mandato de masculinidad y la estructura corporativa de la fratria masculina, verticalista y autoritaria. Este mandato obliga a los hombres a obedecer reglas y jerarquías desde que ingresan a la vida en sociedad y los coloca como las primeras víctimas, imponiéndoles dar pruebas de crueldad y narcisismo permanentemente, primero de forma intragénero y luego hacia las mujeres. Para la expositora, un verdadero cambio cultural que modifique el orden político patriarcal requiere visibilizar el daño que este produce tanto a mujeres como a varones. 


\section{Algunas conclusiones}

Las diversas actividades desarrolladas en el marco del Segundo Congreso Internacional de Victimología contaron con gran presencia y participación de estudiantes de grado y posgrado, profesionales de diversas disciplinas y organizaciones de la comunidad. Esta masiva asistencia y el nivel de los intercambios alcanzados contribuyen a la consolidación en la Facultad de Psicología de la Universidad Nacional de La Plata de un campo de estudio e investigación acerca de las distintas violencias y sus efectos psicosociales.

Cabe destacar la multiplicidad de representaciones que se hicieron presentes en el Congreso provenientes de distintas puntos de Argentina y de otros países, en especial de Latinoamérica, posicionando a nuestra Facultad como una referencia nacional e internacional en la materia.

Los alcances del Congreso trascendieron el ámbito académico a partir de recibir efectores de diversas instituciones estatales y de la comunidad, fortaleciendo así la relación Universidad-comunidad-Estado, contribuyendo a consolidar el lugar de la universidad pública como referencia a la cual acudir en consulta y actualización, siendo a su vez fuente de legitimidad social.

El carácter interdisciplinario e intersectorial del evento se manifestó como una fortaleza del mismo. En este aspecto se destacó como eje central del encuentro la interpelación a los conceptos interdisciplina y equipo interdisciplinario, poniendo en valor los diferentes saberes que habitan las instituciones y las prácticas. Se propuso pensar las problemáticas desde los inter-saberes, habilitando saberes significativos no contenidos por las disciplinas tradicionales, que han sido determinadas por la división del conocimiento característico del paradigma positivista.

Otro aspecto a destacar es la consolidación en los debates de un rol técnico profesional consustanciado con un compromiso social y ético que pone en el centro del interés de la investigación y de la intervención al ser humano, acorde con la perspectiva del paradigma de derechos.

Cabe señalar también que la realización del evento permitió fortalecer el desarrollo e implementación de proyectos de investigación en red, integrando grupos de investigadores/as de diferentes países, promoviendo el trabajo en conjunto. De esta forma, se realizaron articulaciones institucionales con universidades y facultades del exterior para la investigación de problemáticas relacionadas a las violencias.

Para finalizar se destaca como fortaleza institucional la conformación de un equipo de trabajo que permitió llevar adelante el evento aquí reseñado. El mismo se integró con docentes, jóvenes graduados/as, estudiantes y nodocentes de la Facultad de Psicología UNLP constituyendo una organización interclaustros que trabajó con gran compromiso institucional, de manera articulada, con denodado esfuerzo y dedicación para la realización de tan importante Congreso, sin cuyo aporte su realización no hubiera sido posible. 